\title{
Research Paper \\ Investigating the Asymmetric Bimanual Coordination Differences in Male and Female Athletes in Ball and WNon-ball Sports
}

\author{
${ }^{*}$ Yaser Khanjari ${ }^{1}\left(\mathbb{C}\right.$, Elahe Arabameri ${ }^{1}$
}

1. Department of Motor Behavior and Sport Psychology, Faculty of Physical Education and Sport Sciences, University of Tehran, Tehran, Iran.

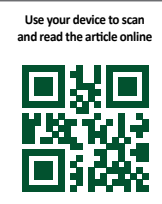

Crtation: Khanjari Y, Arabameri E. [Investigating the Differences of Asymmetrical Bimanual Coordination on Male and Female Athletes WWin Ballplayers and Non-ballplayers (Persian)]. Journal of Sport Biomechanics. 2021; 6(4):250-263. https://doi. org/10.32598/biomechanics.6.3.4

https://doi.org/10.32598/biomechanics.6.3.4

Key words:

Motor coordination, Bilateral interference, Harmonious structures, Eye and hand coordination

\section{A B STRACT}

Objective Bilateral coordination skills are one of the key factors in performing simple to skillful sports task, so far the differences between ball and non-ball fields have not been determined. Therefore, this study aimed to investigate the differences in asymmetric bilateral coordination between male and female athletes in ballplayers and non-ballplayers.

Methods The samples of this study were randomly selected from the young girls and boys (Mean \pm SD of age 26.00 \pm 4.50 ) in ball fields (14) and non-ball fields (16) of the Faculty of Physical Education of the University of Tehran. All subjects had complete visual acuity and the consent form of the research. The instrument of Vienna was used to measure peripheral visual.

Results The statistical results of MANOVA test showed that there is significant difference in asymmetrical bilateral coordination between ballplayers and non-ballplayers and girls and boys $(P \geq 0.05)$.

Conclusion The results of this study showed that asymmetric bilateral coordination performing in ball players had better than non-ball players and girls than boys. According the importance of bimanual coordination skills in the implementation of championship sports, as well as considering gender differences and the positive impact of ball exercises on ball coordination, sports coaches can provide training, especially at a younger age, to facilitate learning of this fundamental skill.

\section{Extended Abstract}

\section{Introduction}

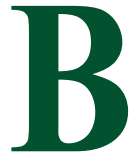

imanual coordination is an important and challenging issue in motor learning needed in daily and recreational activities [3]. An important feature of performing bimanual skills is that both hands tend to do the same thing simultaneously. Sometimes the two hands do not necessarily do the same thing, and each hand acts separately, which is called asymmetric bimanual coordination

[1]. Research shows that the central nervous system easily controls symmetrical movements but has limited control over simultaneous asymmetric movements [6].

Eye-hand coordination plays a significant role in bimanual tasks [20], and this role is more prominent in ball sports (due to constant contact with the ball, catching a ball, and multiple blows to the ball) non-ball sports. On the other hand, athletes in ball and non-ball sports experience various experiences, tools, and environments; therefore, one of the important questions of this study was whether the level of bimanual coordination skills between athletes in ball and non-ball sports is different.

\section{* Corresponding Author:}

Yaser Khanjari, PhD.

Address: Department of Motor Behavior and Sport Psychology, Faculty of Physical Education and Sport Sciences, University of Tehran, Tehran, Iran. Tel: +98 (912) 4551425

E-mail: ykhanjari@ut.ac.ir
} 
No research has been done in this field so far. On the other hand, although most studies have reported gender differences in bimanual coordination skills in favor of girls, there are conflicting results in this regard [26]. This study aimed to investigate the asymmetric bimanual coordination differences in male and female athletes in a ball and non-ball sports.

\section{Methods}

This research was of an applied study with a causal-comparative nature. The number of samples was 30 people with a Mean \pm SD of $26.00 \pm 4.50$ years of age from male and female athlete students of the Faculty of Physical Education, University of Tehran, who were randomly selected. In this study, to control the subjects' level of experience, athletes active in the relevant sport at the semi-professional and professional levels were selected. Finally, the research samples were divided into two groups, with 14 people in the ball sports group (ball-related sports) and 16 people in the non-ball sports group (sports that do not require a ball or special tools).

It should be noted that ball sports included football, futsal, basketball, handball, volleyball, and table tennis, and nonball sports included wrestling, karate, taekwondo, swimming, and track-and-field. Also, most of the subjects had a history of professional sports at the provincial and national team levels. To measure asymmetric bimanual coordination, the Vienna Test System was used, which was available in the "learning and movement control laboratory" of the Faculty of Physical Education, University of Tehran. All subjects completed the consent form for voluntary participation in the research and the physical health form.

Kolmogorov-Smirnov (K-S) test was used for normal data distribution, and Levene's test was used to assess the equality of variances of the groups. MANOVA statistical method was used to test the research hypothesis, and also Box's $M$ test was used to equalize the covariance matrix of the groups. A significance level of $\mathrm{P} \leq 0.05$ was considered for data analysis.

\section{Results}

In Table 1, the performance time (speed) and error time (accuracy) of the research subjects were presented separately for male and female athletes in the ball and nonball sports. These results confirm that ball sports athletes spent less total time on the task. Also, ball sports' athletes had less total error time performing the task; that is, they had higher accuracy than non-ball sports' athletes. Performance time by female athletes (with a mean of $28.83 \mathrm{sec}$ onds) and male athletes (with a mean of 38.94 seconds) indicated that girls had higher speed and better performance in performing the tasks. Also, in examining the female athletes' error time (with a mean of 0.34 seconds) and male athletes (with a mean of 0.61 seconds), it can be concluded that girls had fewer errors and had a higher degree of accuracy in performing bimanual coordination task.

MANOVA statistical results indicated that there was a significant difference between girls and boys in the dependent variables of "performance time" (speed) and "error time" (accuracy) $(\mathrm{P} \leq 0.05)$. There was also a significant difference between ball and non-ball sports athletes in these dependent variables $(\mathrm{P} \leq 0.05)$. Finally, the statistical results of this study revealed that the interaction effect of the two variables of "ball and non-ball" and "gender" on the dependent variables of "performance time" and "error time" was not significant $(\mathrm{P} \geq 0.05)$. In general, the results of this study confirmed that in performing asymmetric bimanual coordination tasks, ball sports' athletes performed better than nonball sports' athletes, and girls performed better than boys.

\section{Discussion and Conclusion}

One of the results of this study was that girls had a significant advantage over boys in performing asymmetric bimanual coordination tasks. It seems that one of the reasons for the superiority of girls' performance over boys in this study was related to performing a delicate task that required the use of delicate muscles of the fingers with high accuracy. Also, the results of some studies by MRI showed that the area of the corpus callosum in girls was larger than boys, as a result of which the exchange of information between the two hemispheres of the brain was more accessible, which makes girls superior to boys in performing bimanual coordination tasks [30].

The better performance of ball sports athletes compared to non-ball sports athletes in performing asymmetric bimanual coordination skills was another result of this study, which can be related to years of practice and experience working with both hands and balls in the ball sports group. Also, the task arranged in this study required high coordination between hand and eye movements, which in various studies it has been confirmed that ball exercises have a positive effect on the eye and hand coordination [41]; therefore, one of the possible reasons for the superiority of the bimanual coordination skills of the ball group can be attributed to the stronger coordination between eyes and. Finally, due to the high importance of bimanual coordination skills in sports success [43], it is expected that sports coaches, especially in the education of younger ages, pay attention to this critical issue. 
Table 1. Mean performance time and mean error time in the ball and non-ball sports athletes

\begin{tabular}{|c|c|c|c|c|}
\hline Dependent Variable & Gender & Status & Mean $\pm S D$ (seconds) & No. \\
\hline \multirow{9}{*}{$\begin{array}{l}\text { Mean performance time } \\
\text { (speed) }\end{array}$} & & Ball & $25.56 \pm 5.43$ & 7 \\
\hline & Female & Non-ball & $31.69 \pm 10.81$ & 8 \\
\hline & & Total & $28.83 \pm 9.01$ & 15 \\
\hline & & Ball & $33.61 \pm 10.80$ & 7 \\
\hline & Male & Non-ball & $43.61 \pm 4.14$ & 8 \\
\hline & & Total & $38.94 \pm 9.23$ & 15 \\
\hline & & Ball & $29.58 \pm 9.21$ & 14 \\
\hline & Total & Non-ball & $37.65 \pm 10.02$ & 16 \\
\hline & & Total & $33.89 \pm 10.33$ & 30 \\
\hline \multirow{9}{*}{ Mean error time (accuracy) } & & Ball & $0.28 \pm 0.18$ & 7 \\
\hline & Female & Non-ball & $0.40 \pm 0.19$ & 8 \\
\hline & & Total & $0.34 \pm 0.19$ & 15 \\
\hline & & Ball & $0.53 \pm 0.21$ & 7 \\
\hline & Male & Non-ball & $0.69 \pm 0.14$ & 8 \\
\hline & & Total & $0.61 \pm 0.19$ & 15 \\
\hline & & Ball & $0.40 \pm 0.23$ & 14 \\
\hline & Total & Non-ball & $0.54 \pm 0.22$ & 16 \\
\hline & & Total & $0.48 \pm 0.23$ & 30 \\
\hline
\end{tabular}

\section{Ethical Considerations}

Compliance with ethical guidelines

All ethical principles are considered in this article. The participants were informed of the purpose of the research and its implementation stages. They were also assured about the confidentiality of their information and were free to leave the study whenever they wished, and if desired, the research results would be available to them. A written consent has been obtained from the subjects. The study was performed in accordance with the Helsinki Convention.

\section{Funding}

The paper was extracted from a research project of the Dr. Yaser Khanjari \& Dr. Elahe Arabameri, Department of motor behavior and sport psychology, Faculty of Physi- cal Education and Sport Sciences, University of Tehran, Tehran, Iran.

\section{Authors' contributions}

All authors equally contributed to preparing this article.

\section{Conflicts of interest}

The authors declared no conflict of interest.

\section{Acknowledgements}

We would like to thank all the students of the Faculty of Physical Education, University of Tehran and the Experts of the Learning and Movement Control Laboratory who helped us in this research. 


\title{
كى تفاوتهاى هماهنتى دو دستى نامتقارن در ورز شكار ان دختر و يسر رشتهاى توبى وغيرتوبى
}

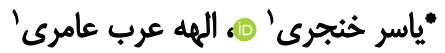

ا. كروه رفتار حركتى و روانشناسى ورزشى، دائشكدة تربيتبدنى وعلومورزشى، دانشكاه تهران، تهران، ايران.

\begin{abstract}
حكيد

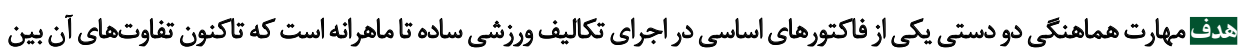

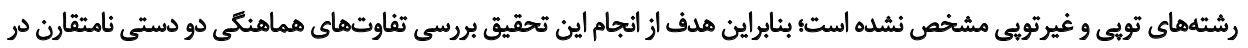

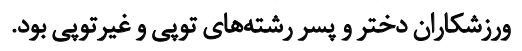

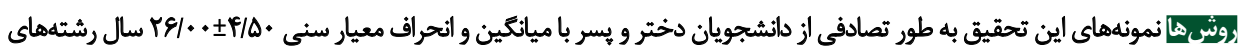

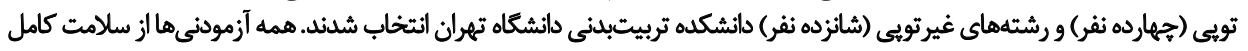

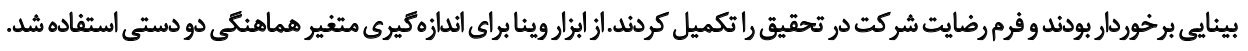

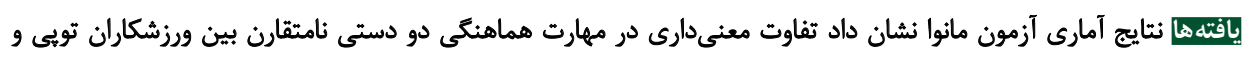

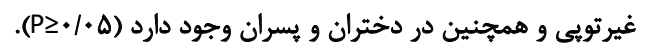

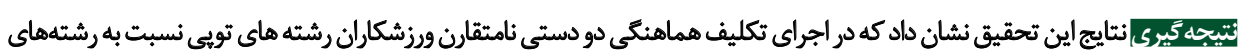

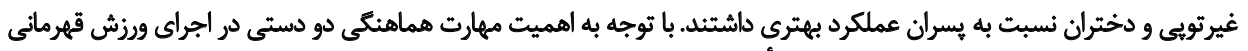

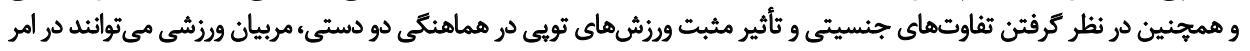

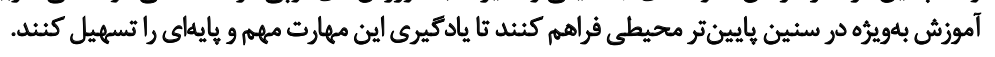

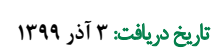

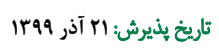

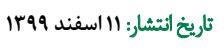

ويرُكى مههم اجراى مهارتهاى دو دستى اين است كه دو داري

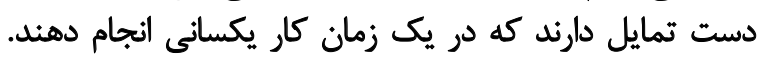

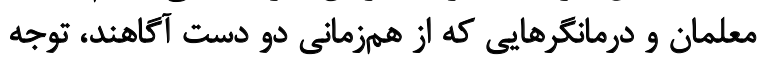

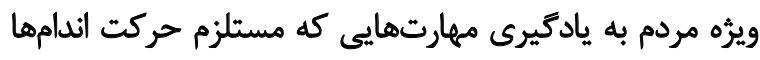

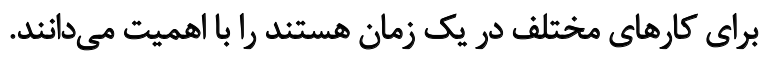

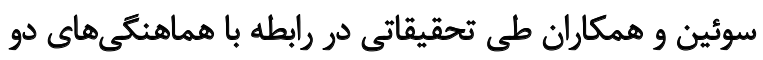

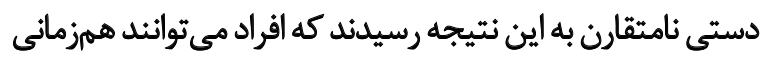

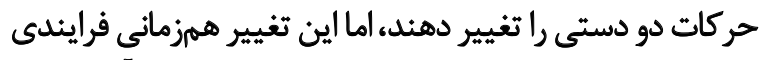

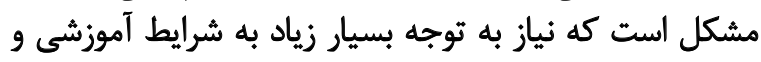

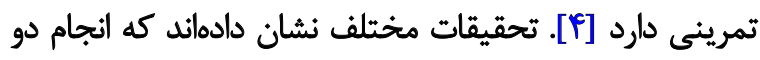

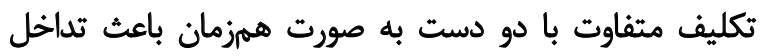

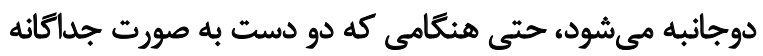

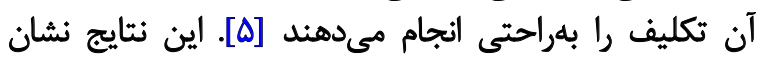

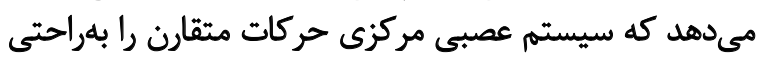

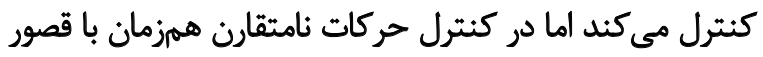

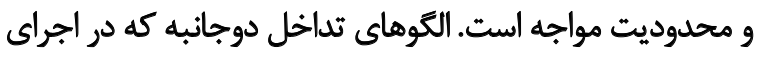
تكاليف هماهنگى دو دستى نامتقارن اتفاق مى افتد تمايل دورايل شديد دراي

بسيارى از حركات ماهرانه انسان نياز به همكارى دو دست دارد.

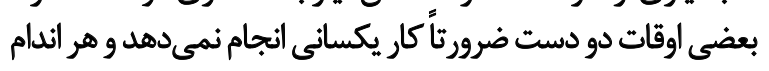

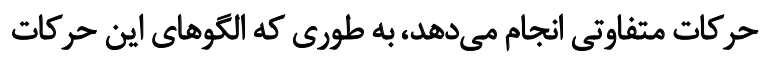

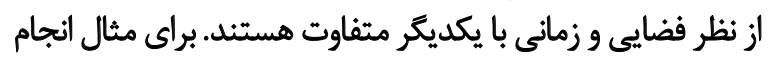

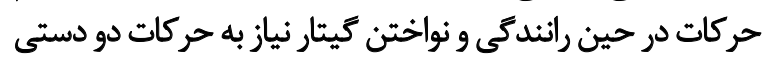

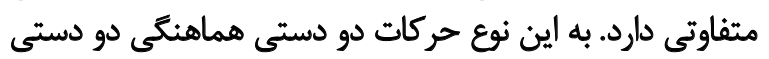

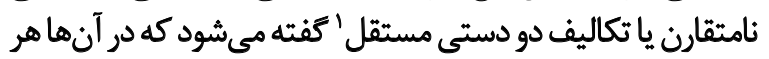

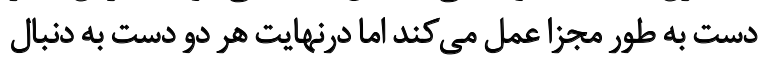

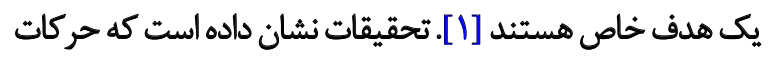

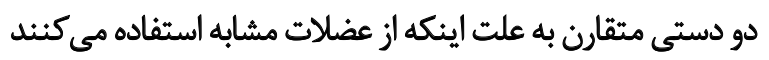

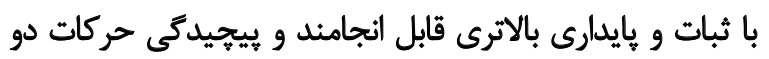

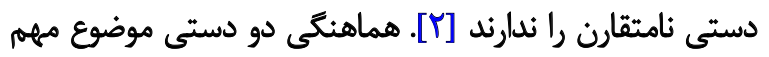

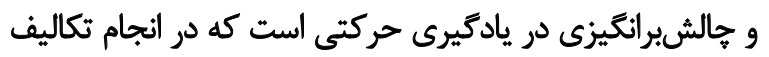

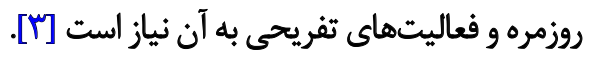

1. Independent bimanual tasks

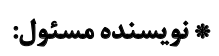

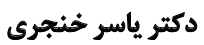

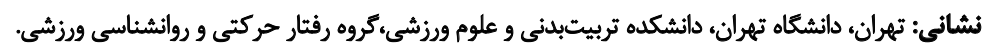

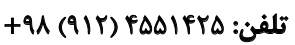
يست الكترونيكي: ykhanjari@ut.ac.ir 
با هم (هم كوشى ها)ه در قالب همكارى يك گروه ويرها از عضلات

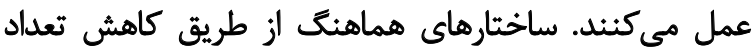

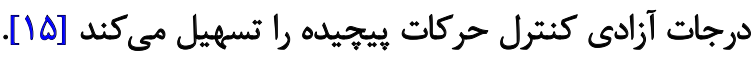

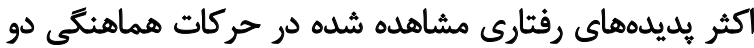

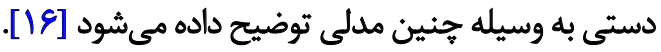

در سالهاى اخير علاقه محققان و متخصصان ورزشى به به بهائ بررسى توانايى هماهنكى حركتى افرئي افراد بيشتر شده است است زيران

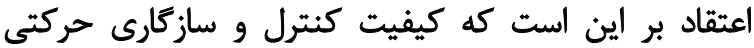

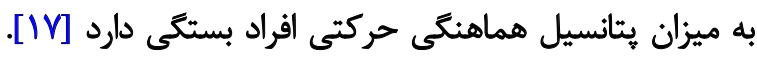

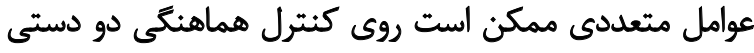

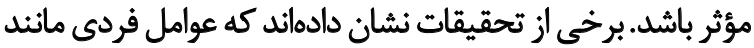

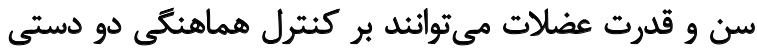

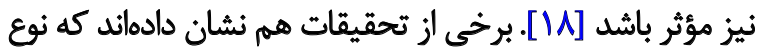

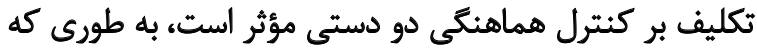

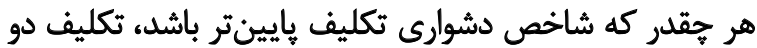

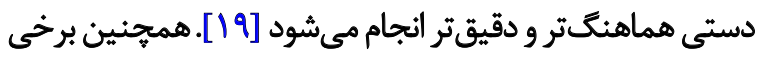

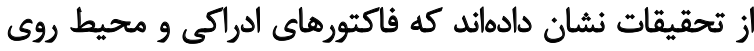

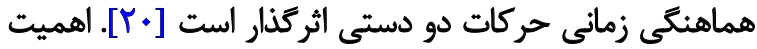

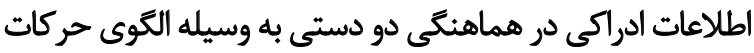

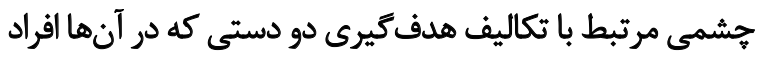

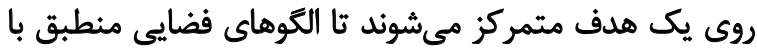

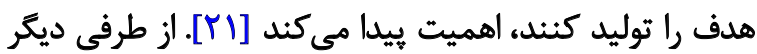

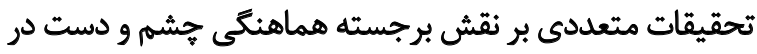

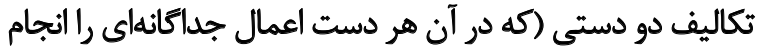

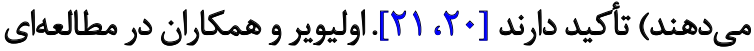

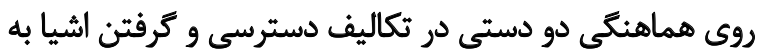

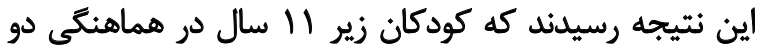

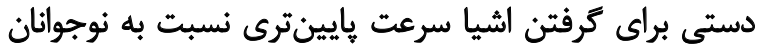

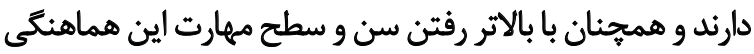

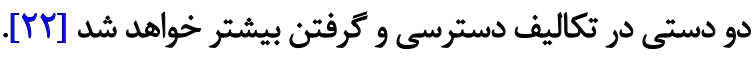

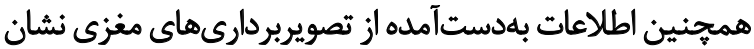

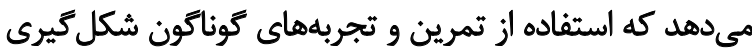

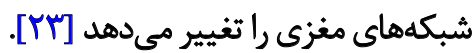

با توجه به نقش مهم هماهنكى خشم و دست در كرفتن و

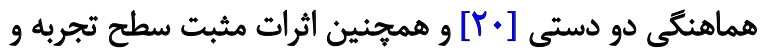

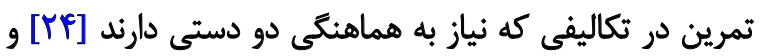

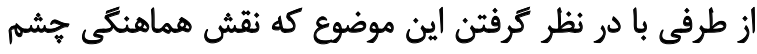

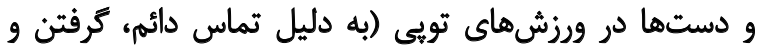

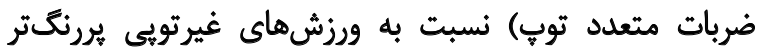

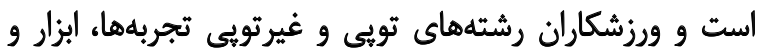

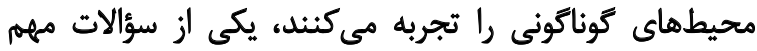
تحقيق حاضر اين است كه آيا سطح مهارت هماهنئكى دو دستى
سيستم عصبى مركزى را در اجراى حركات متقارن، مشابه و يا

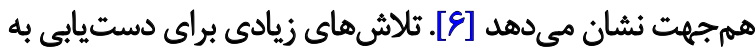

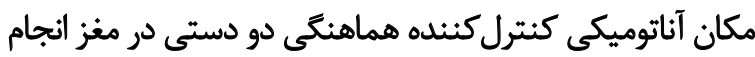

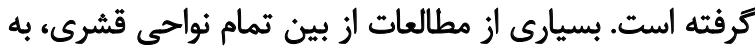

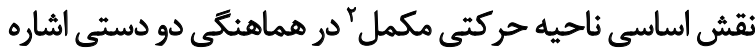

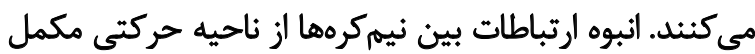

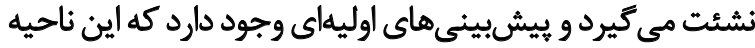

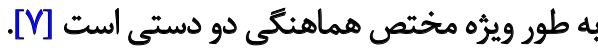
برخي تحقيقات نشان داداند كه فعاليت عصبى در ناحيه

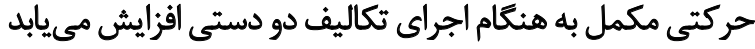

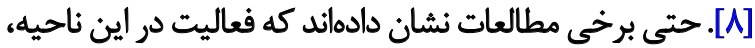

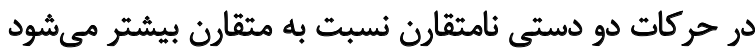

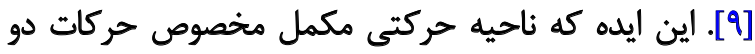

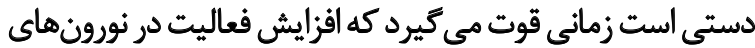

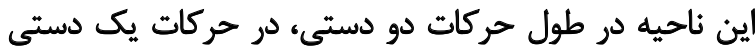

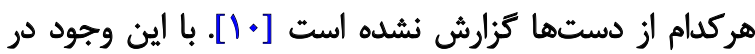

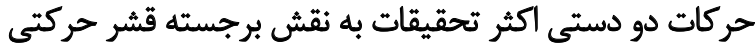
نيم كره دكرسوى دست برتر نيز اشاره داشتهاند [ [11]. تمايل شديد دو دست براى توليد حركات مشابه از نظر فضايى دراني

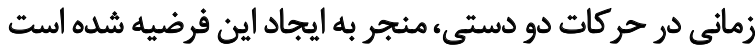

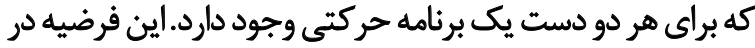

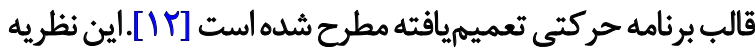

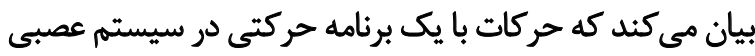

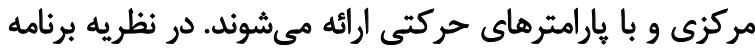

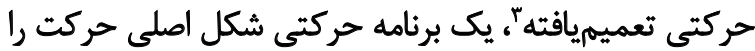

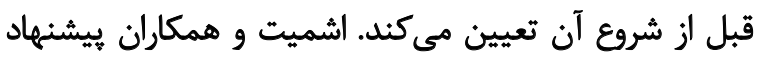

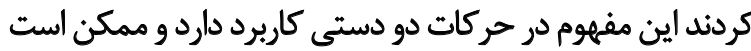

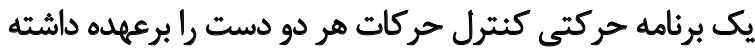

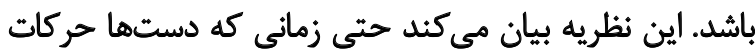

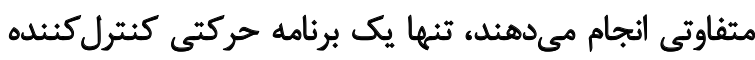

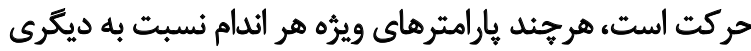

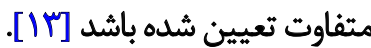

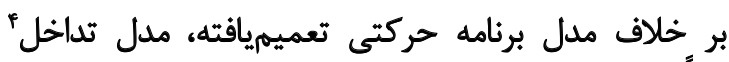

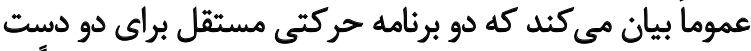

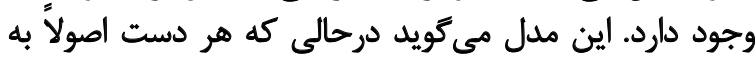

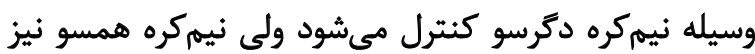

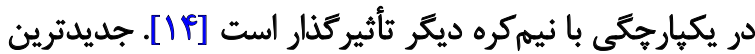

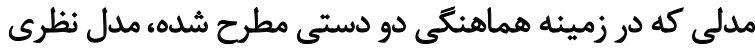

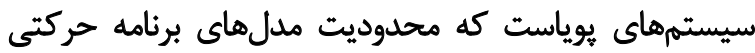
تعميميافته و تداخل راندارد. در اين مدل ساختار هاى هماهنى برديت

2. Supplementary Motor Area

3. Generalized Motor Program

4. Crosstalk model 
هماهنكى دو دستى نامتقارن از ابزار وينا موجود در آزمايشكاه

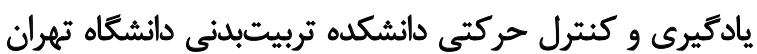
استفاده شد. - ادي در ابتدا همه آزمودنىها فرم رضايت داوطلبانه شركت در

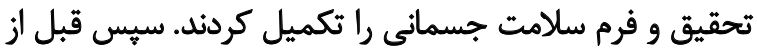

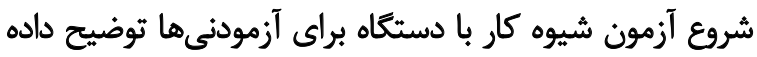

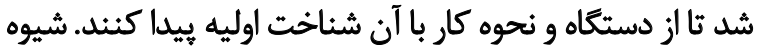

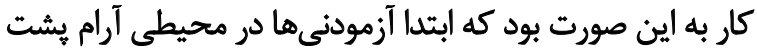

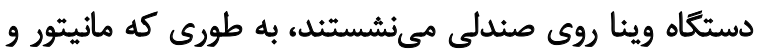

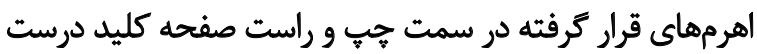

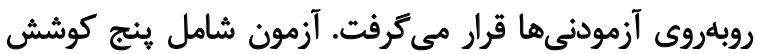

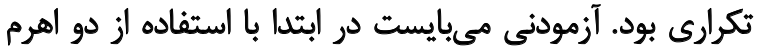

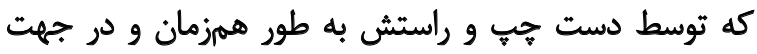

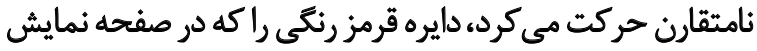

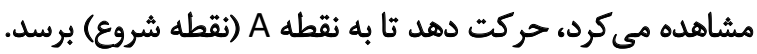

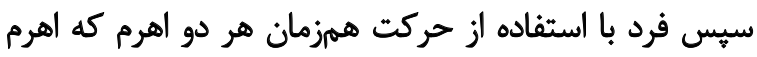

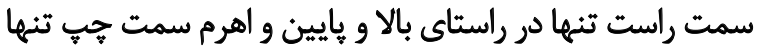

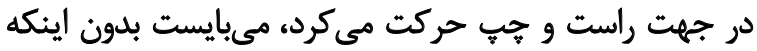

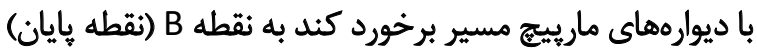

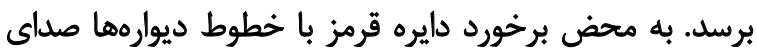

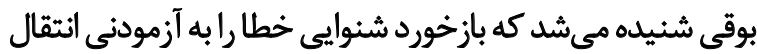

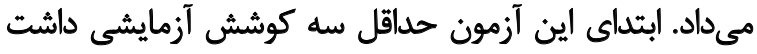

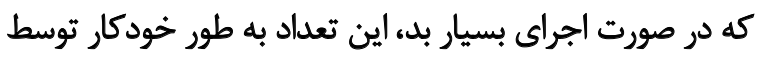

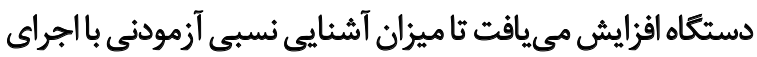

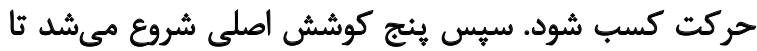
دستكاه بيام خاتمه آزمون را العلام كند.

ميس از اجراى آزمون دستعاه نتايج آزمون افراد را بر اساس

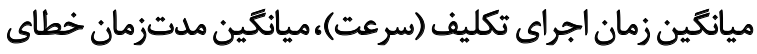

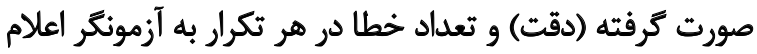

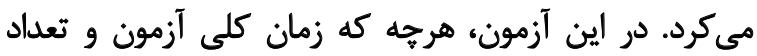

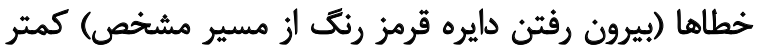

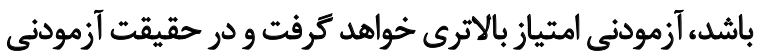
عملكرد بهترى داشته است (تصوير شماره () ). براى آمار توصيفى دادهها از ميانكين و انحراف معيار استفاده

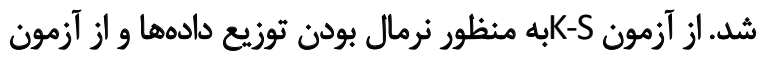

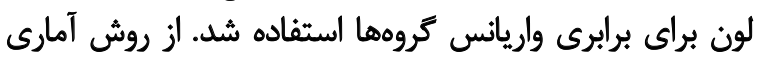

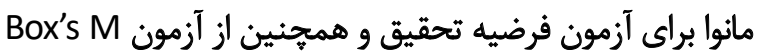

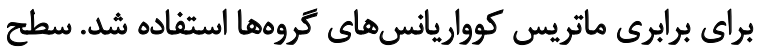

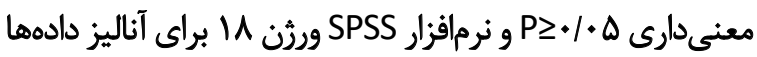
در نظر كرفته شد.

6. Vienna Test System
بين ورزشكاران تويى و غيرتويى متفاوت است؟ ناكنون تحقيقى إئى

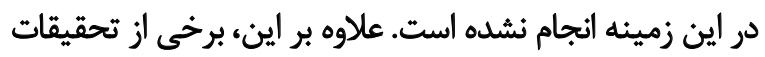

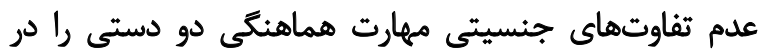

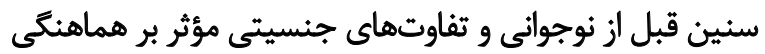

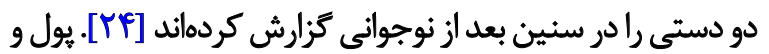

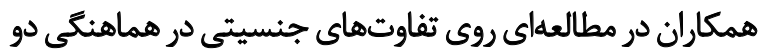

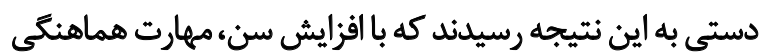

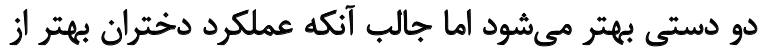

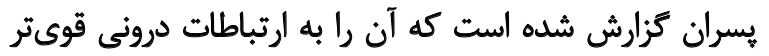

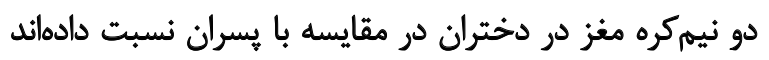

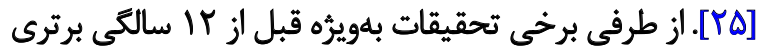

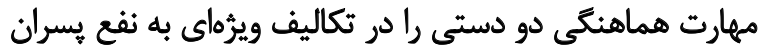

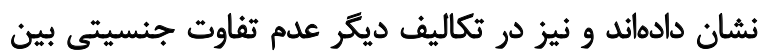

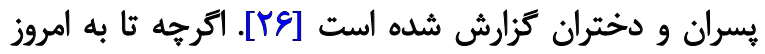

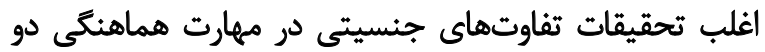

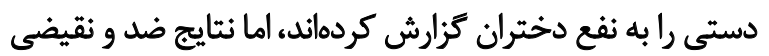

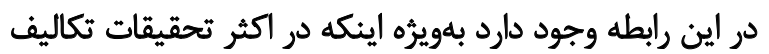

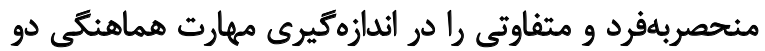

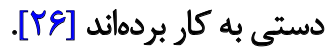

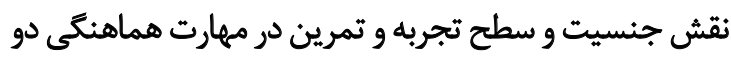

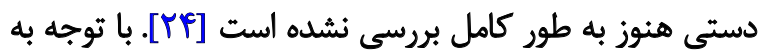

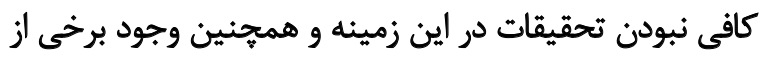

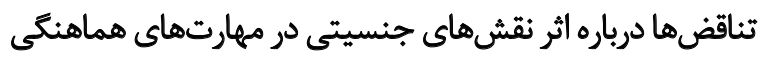

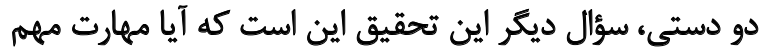

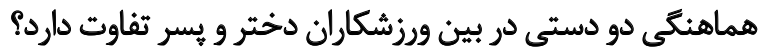

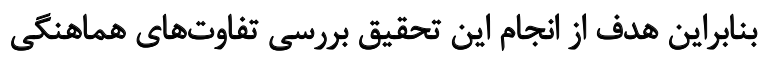

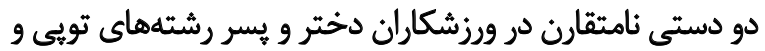
عُيرتويى بود. روشُشُشى تحقيق حاضر از نوع كاربردى با ماهيت علّي مقايسهاى است.

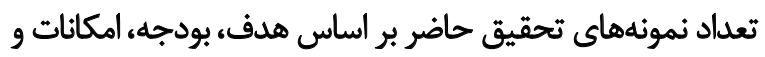

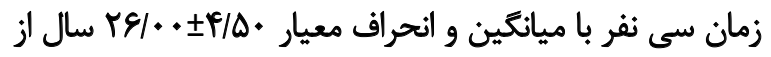

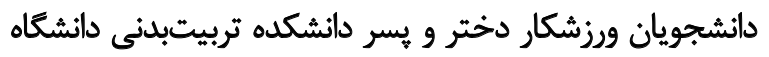

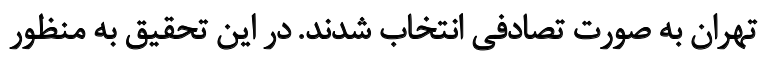

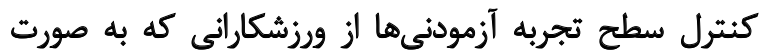

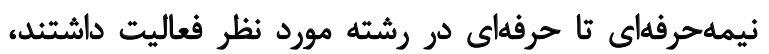

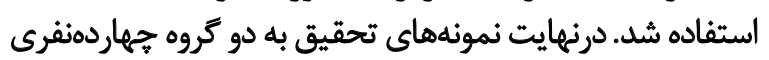

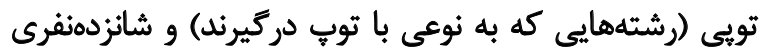

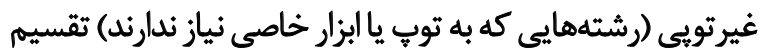

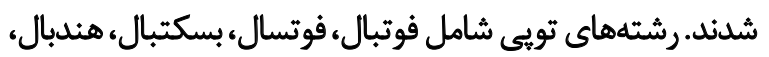

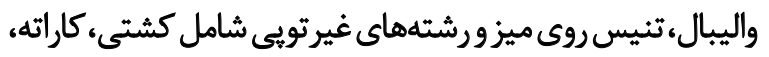

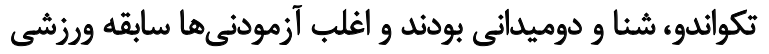
حرفهاى (استانى تا تيم ملى) را داشتند. به منظور اندازهكيرى 


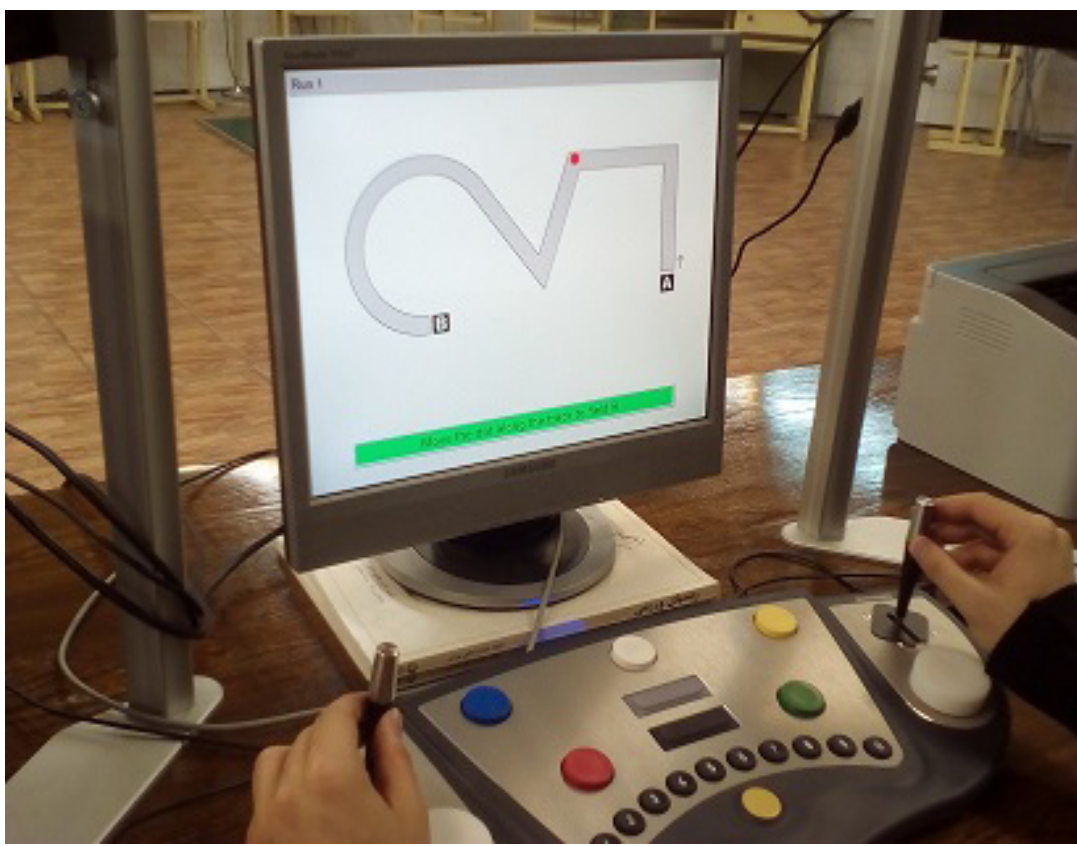

اجرا (سرعت) و مدتزمان خطا (دقت) وجود داشت (ه (P> (P).

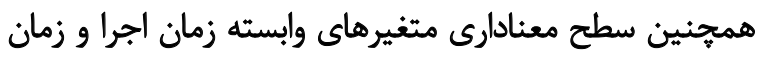

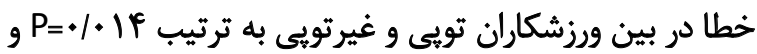

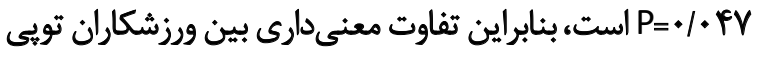

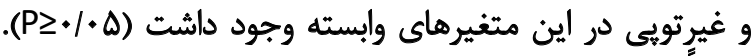

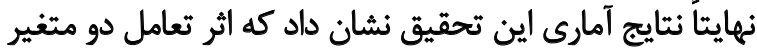

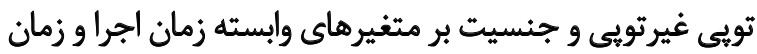

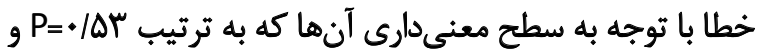

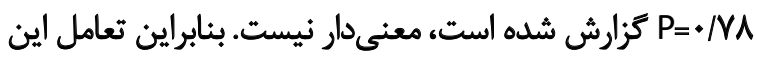

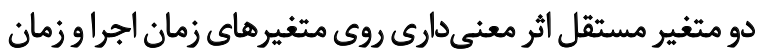

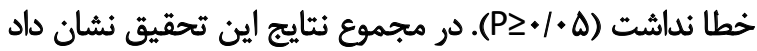

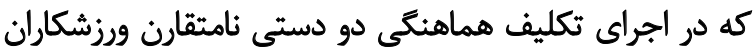

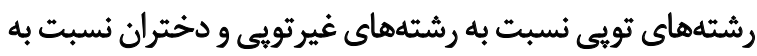
يسران عملكرد بهترى داشتئد.

بحث

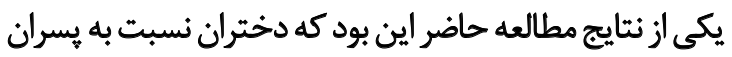

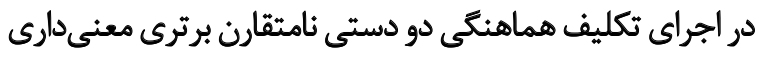

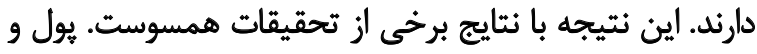

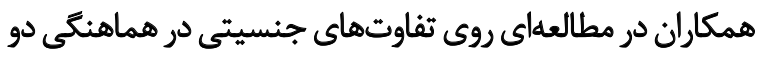

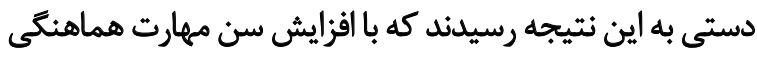

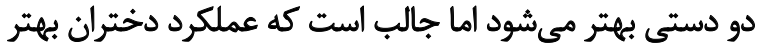

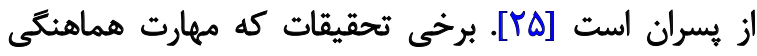

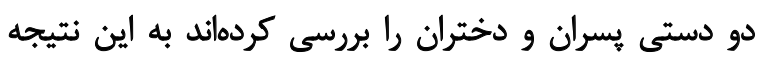

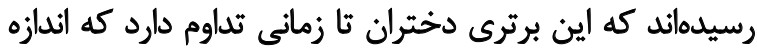
انكُشتان اشاره و شست هر دو كروه يكسان باشد. در مقابل برخى تراني
ثنايج

جدول شماره المار توصيفى مربوط به زمان اجرا (سرعت) (آمون

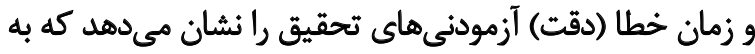

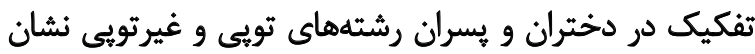

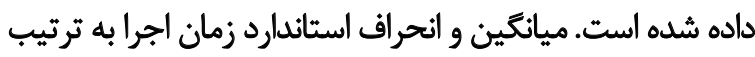

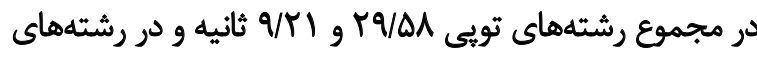
غيرتويى

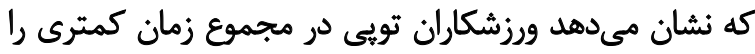

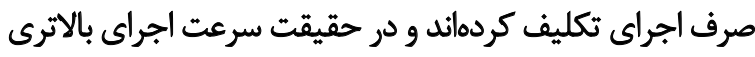

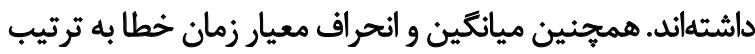

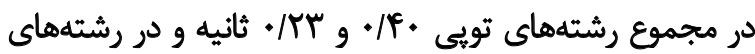

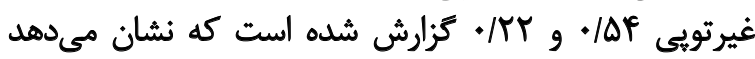

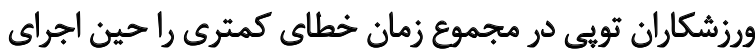

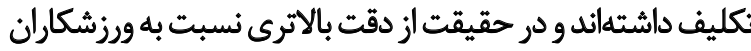

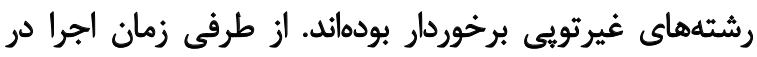

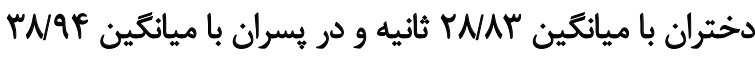

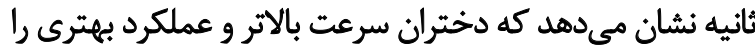

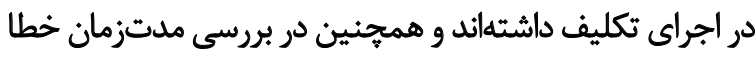

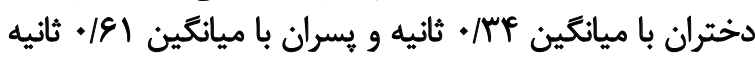

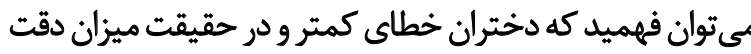

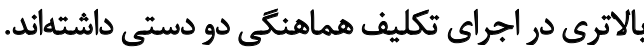

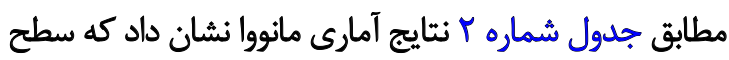

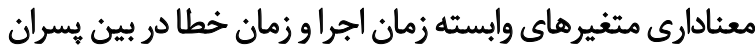

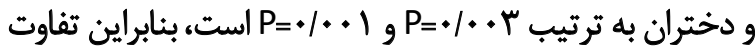

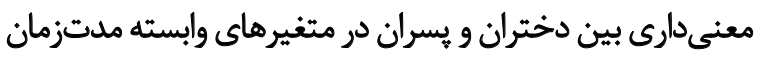


جدول اـ آمار توصيفى ميانكين زمان اجراو زمان خطا در ورزشكاران تويى و غيرتويى

\begin{tabular}{|c|c|c|c|c|}
\hline تعداد & ميانَّين土|نحراف استاندارد (ثانيه) & تويى و غيرتويى & جنسيت & متغير وابسته \\
\hline$r$ & $r \Delta / \Delta F \pm \Delta / \mu r$ & وزشكاران تويى & & \multirow{9}{*}{ هيانكين زعان اجرا } \\
\hline$\wedge$ & $\mathrm{r} / / 99 \pm 1 \cdot / \wedge 1$ & ورزشكاران غيرتويى & لدختر & \\
\hline 10 & TNAYIV・1 & مجموع & & \\
\hline 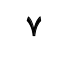 & $m / \varepsilon \mid \pm 1 \cdot / \lambda$. & ورزشكاران ثويى & & \\
\hline$\wedge$ & $r r / \varepsilon \mid \pm F / / F$ & ورزشكاران غيرتويى & 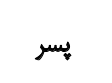 & \\
\hline 10 & rNATIVMT & مجمو & & \\
\hline if & TV/AU士Q/TI & ورزشكاران تويى & \multirow{3}{*}{ مجمقع } & \\
\hline 18 & $r V / \& \Delta \pm 1 \cdot 1 \cdot r$ & ورزشكاران غيرتويى & & \\
\hline r. & $\pi / / \Lambda 9 \pm 1 \cdot / \mu r$ & مجموع & & \\
\hline 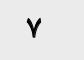 & $. / T A \pm \cdot M A$ & ورزشكاران تويتى & \multirow{3}{*}{ ن دختر } & \multirow{9}{*}{$\begin{array}{c}\text { ميائكين زمان خُان } \\
\text { (دقات }\end{array}$} \\
\hline$\wedge$ & $\cdot / 4 \cdot \pm \cdot / 19$ & ورزشكاران غيرتويى & & \\
\hline 10 & $. / m \pm \pm \cdot / 19$ & مجموع & & \\
\hline v & $+|\Delta H \pm+/ M|$ & ورزشكاران تويى & & \\
\hline$\wedge$ &. $\mid 89 \pm \cdot / f F$ & ورزشكاران غيرتويى & 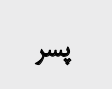 & \\
\hline 10 & $. / 81 \pm . / 19$ & مجموع & & \\
\hline if & $\cdot / \varphi \cdot \pm \cdot / \pi$ & وزيكاران تويى & & \\
\hline is & $\cdot / \Delta F \pm / R r$ & ورزشكاران غيرتويى & مجموع & \\
\hline r. & ./ץA土./r & مجموع & & \\
\hline
\end{tabular}

مجله بيومكانيك ورنش

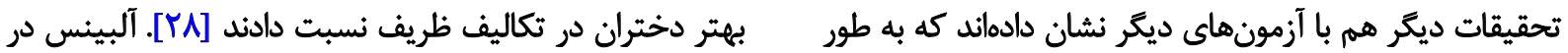

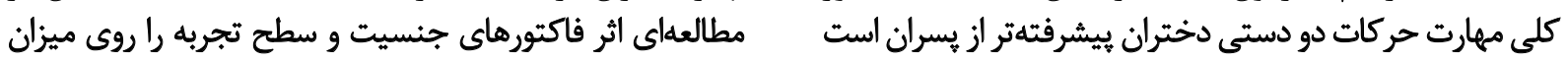

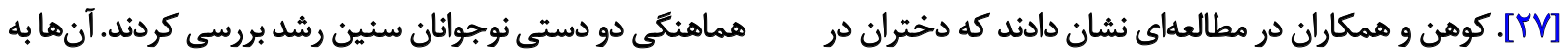

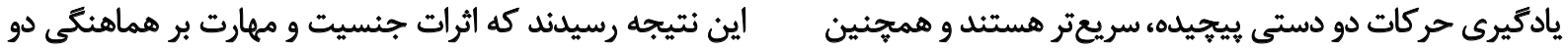

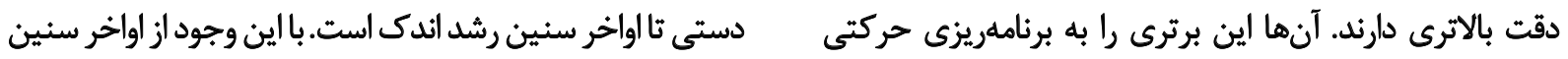

جدول ז. نتايج آزمون مانووا بروى متغيرهاى زمان اجراو زمان خطا در تكليف هماهنكى دودستى نامتقارن

\begin{tabular}{|c|c|c|c|c|c|}
\hline $\mathbf{P}$ & $\mathbf{F}$ & Mean Square & دوجه آزادى & مثغير وابسته & مثغير مستقل \\
\hline $.0 \%$ & 1.180 & $n e t / v$. & 1 & ميائكين زمان اجرا (سرعت) & \multirow{2}{*}{ جئسيت } \\
\hline $.1+1$ & $\mid \omega / N$ & ./AT & 1 & ميانكين زمان خطا (دقت) & \\
\hline .1 .18 & s/af & PAA/AP & 1 & ميانكين زمان اجرا (سرعت) & \multirow{2}{*}{ تويى و غيرتويى } \\
\hline $.1 \cdot \mathrm{pq}$ & $\mathbb{P} / M^{\prime}$ &.$/ 16$ & 1 & ميانكين زمان خطا (دقت) & \\
\hline.$/ \Delta r$ & $+/ 4$ & $r N+1$ & 1 & ميانكين زمان اجرا (سرعت) & \multirow{2}{*}{ جنسيت × تويى و غيرتويى } \\
\hline . $\mathrm{NA}$ & $.1 \cdot V$ & $.10 .+r$ & 1 & ميانكين زمان خطا (دقت) & \\
\hline
\end{tabular}


ناحيه جسم بينهاي در دختران است. همجنين برخى مطالعات

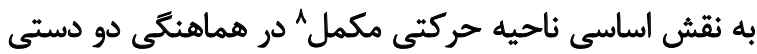

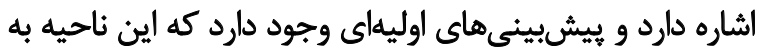

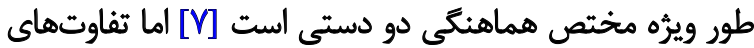

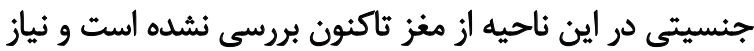

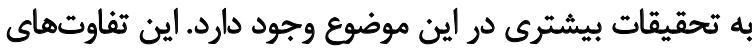

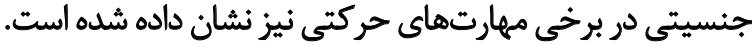

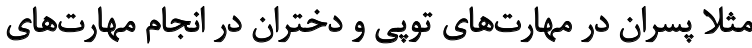

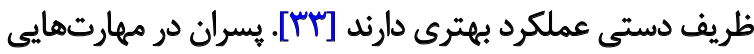

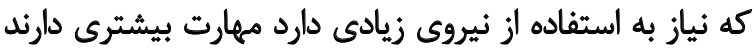

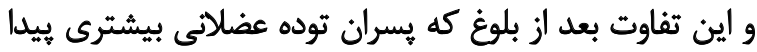

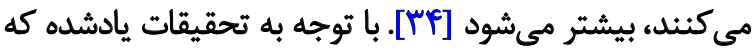

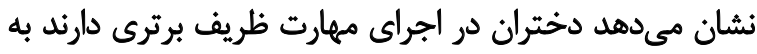

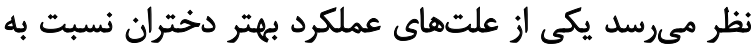

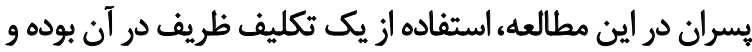

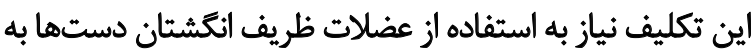

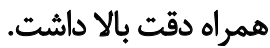

يكى ديكر از نتايج اين مطالعه اين بود كه ورزشكاران رشتهاي

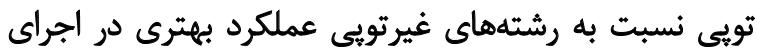

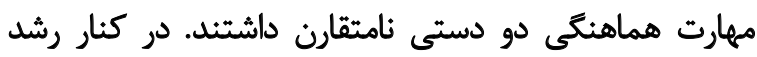

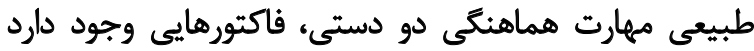

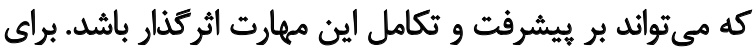

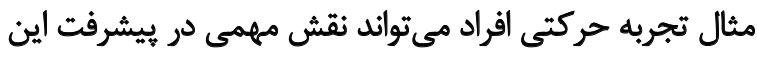

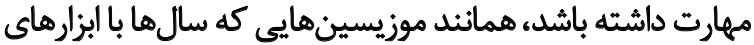

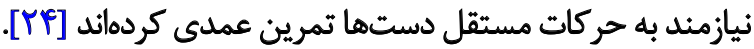

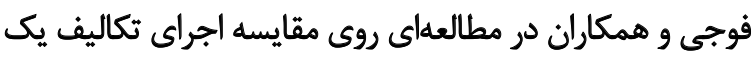

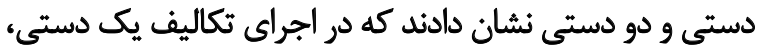

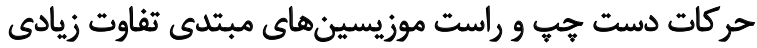

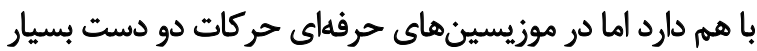

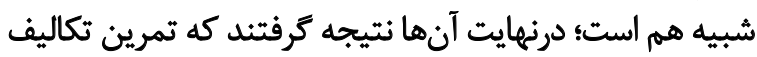

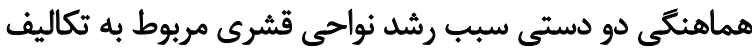

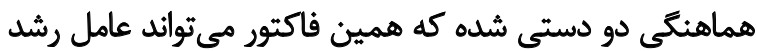

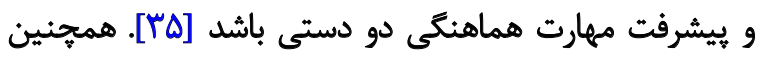

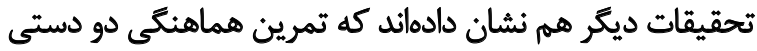

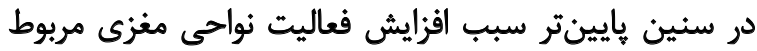

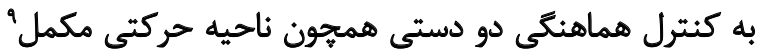

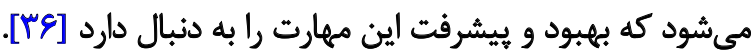

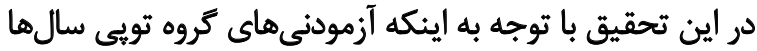

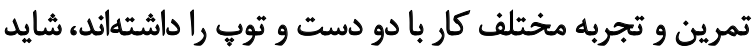

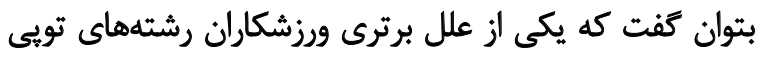

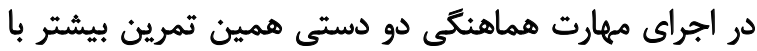

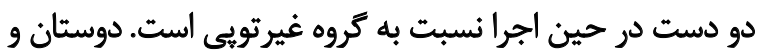

8. Supplementary motor area

9. Supplementary motor area
نوجوانى به بعد تفاوتهاى جنسيتى در هماهنكى دو دستى كه

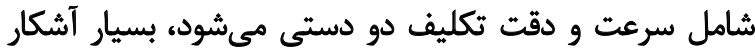

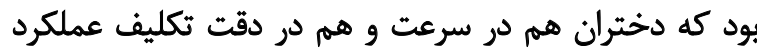

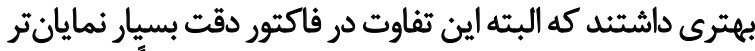

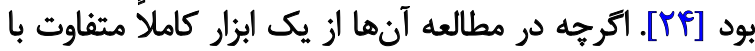

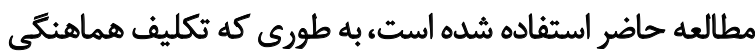

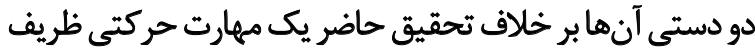

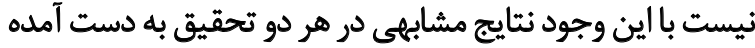

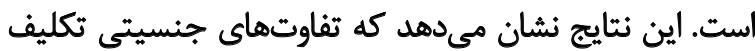

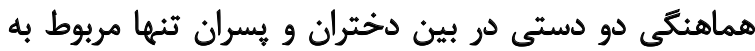

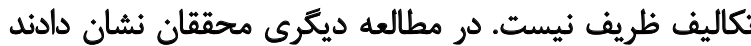

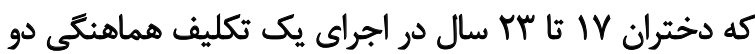

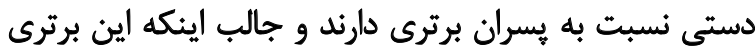

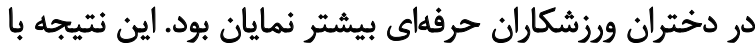

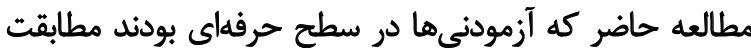

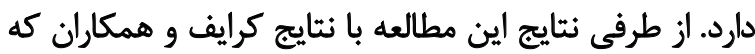

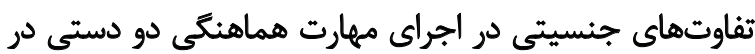

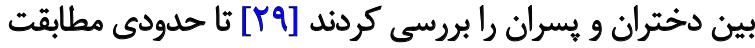

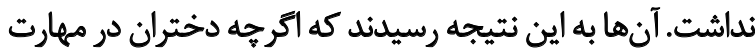

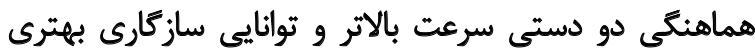

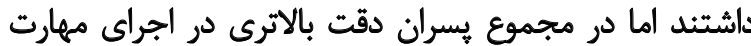

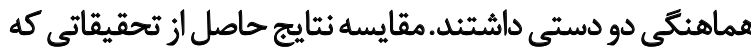

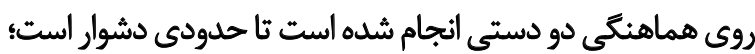
زيرا هركدام از اين تحقيقات از ابزار متفاوتى براي انداني اندازهيري

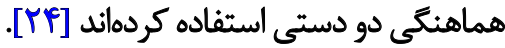

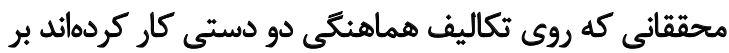

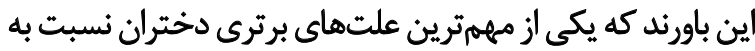

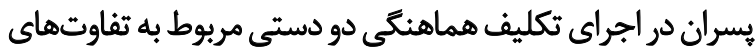

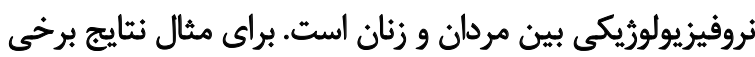

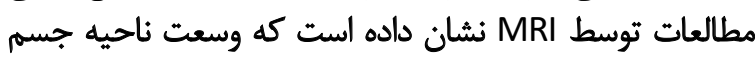

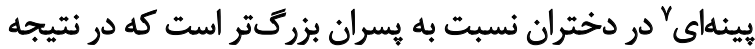

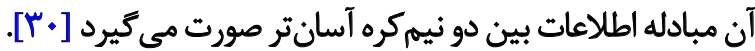

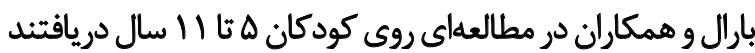

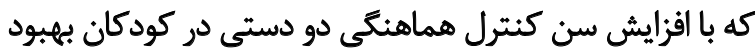

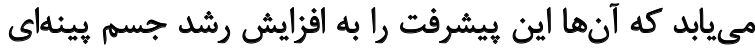

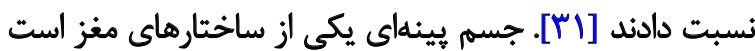

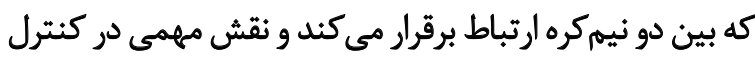

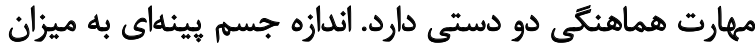

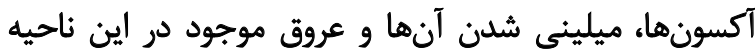

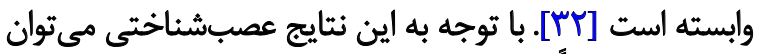

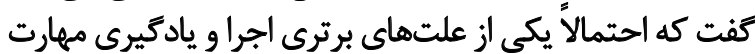

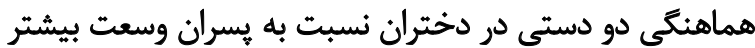


كه الكَوى هماهنكى جشم و دست فاكتورى مهم در ماهر شدن در

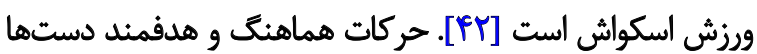

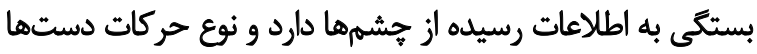

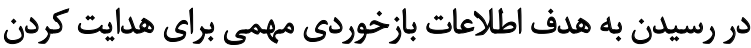

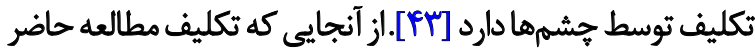

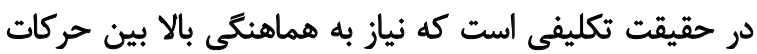

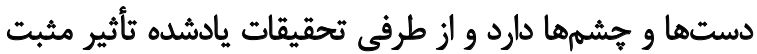

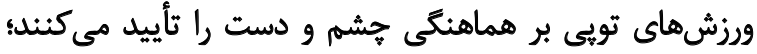

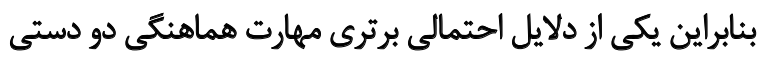

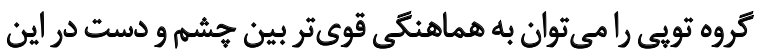

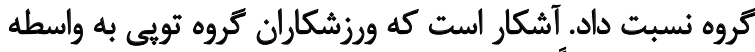

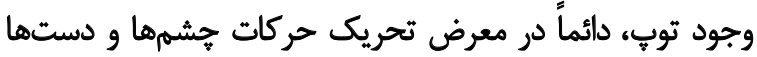

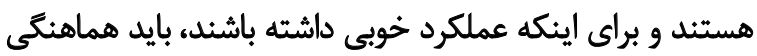

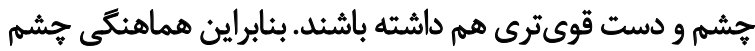

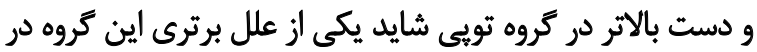
اجراي مهارت هماهنكى دو دستى باشد.

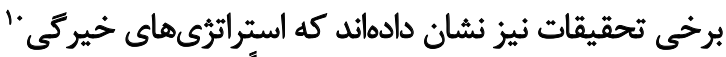

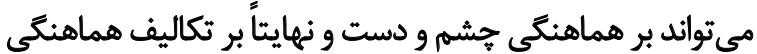

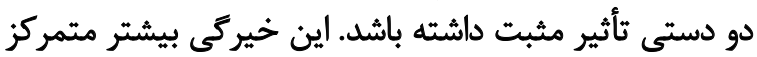

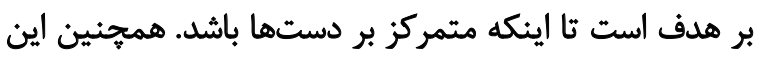

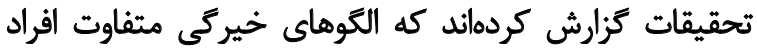

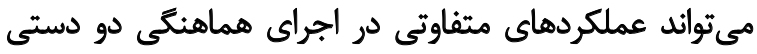

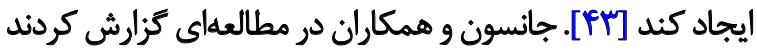

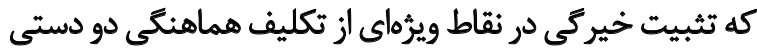

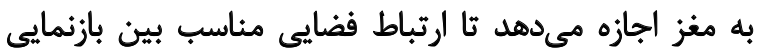

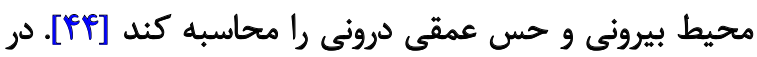

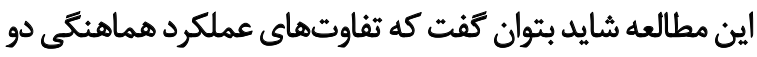

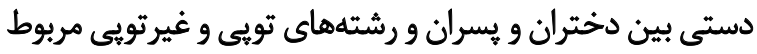

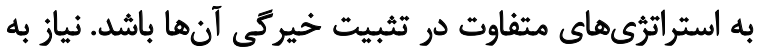

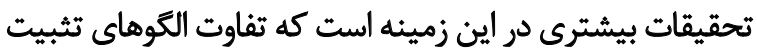

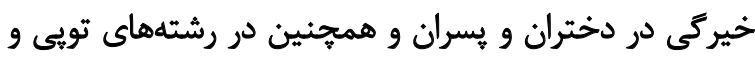

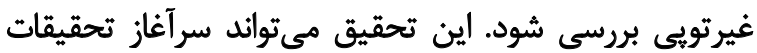

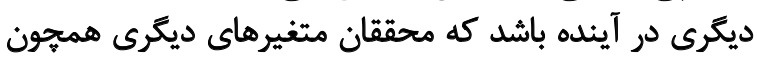

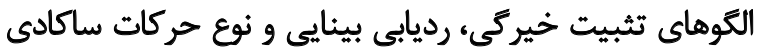

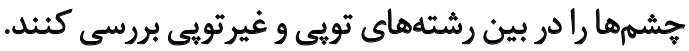

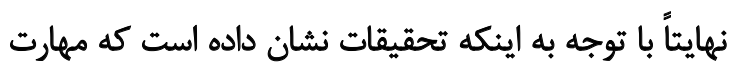

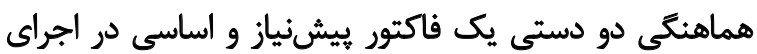

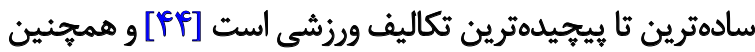

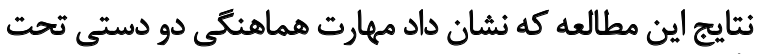

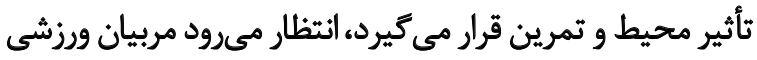

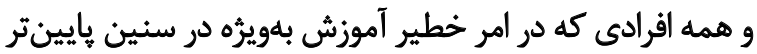

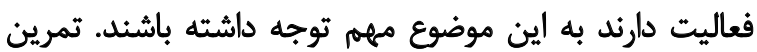

10. Gaze strategies
همكاران در مطالعهاى انتقال حركت دو دستى نامتقارن به حالت

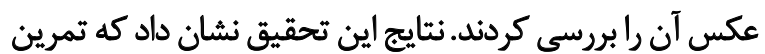

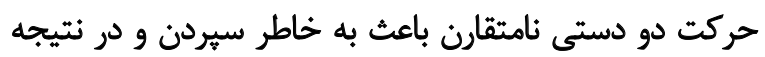

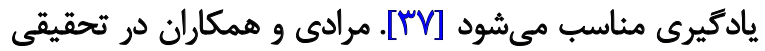

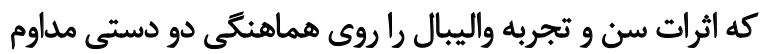

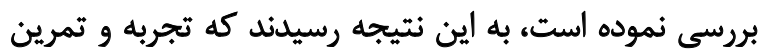

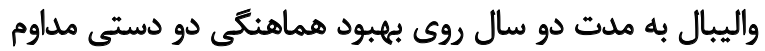

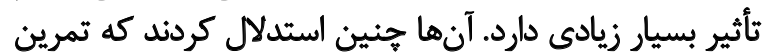

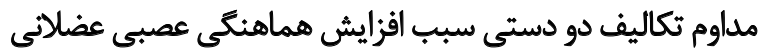

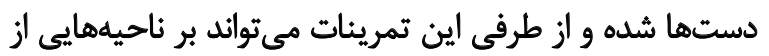

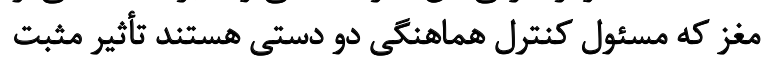

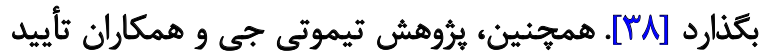

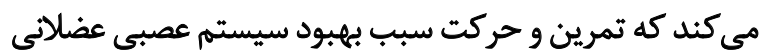

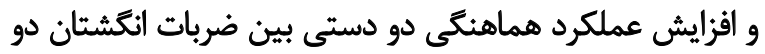

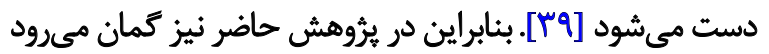

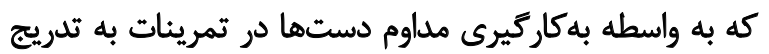

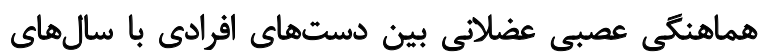

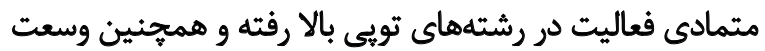

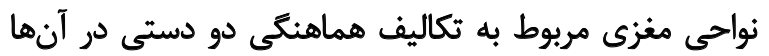

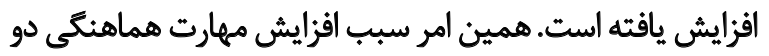

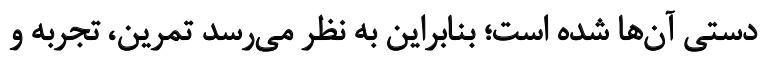

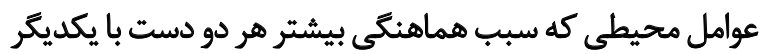

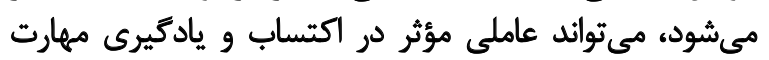

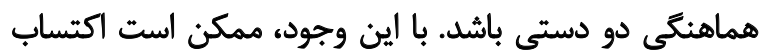

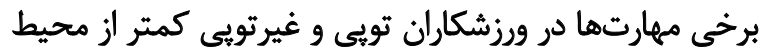

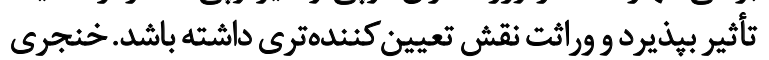

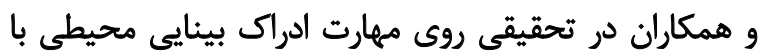

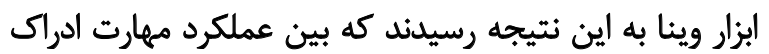

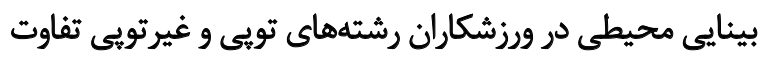

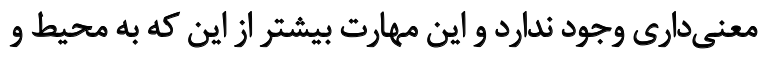

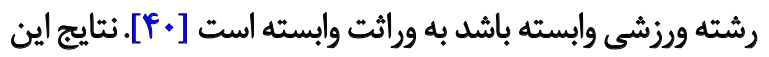

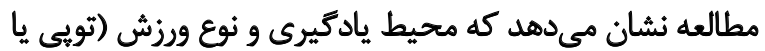

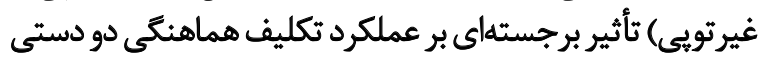

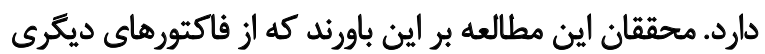

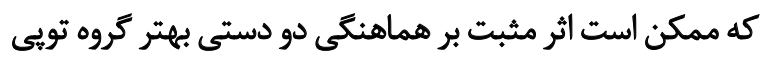

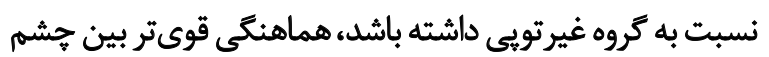

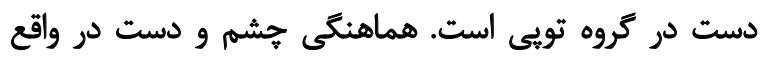

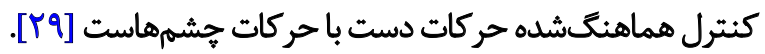

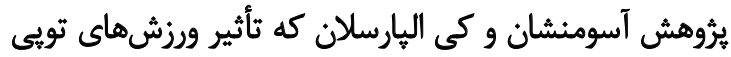

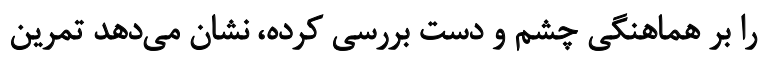

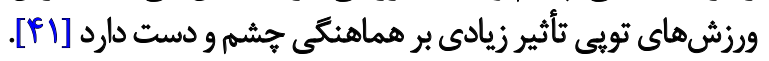

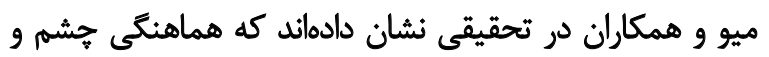

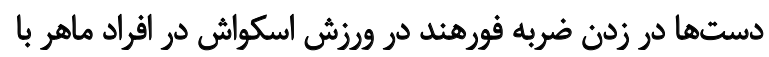

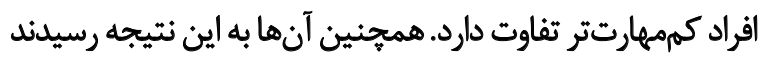


مهارتهاى هماهنكَى دو دستى در سنين هايينتر از طريق

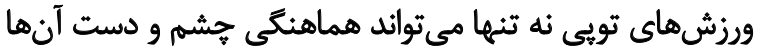

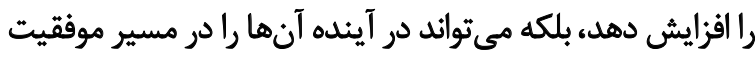
رشتههاي ورزشى كه نياز به هماهنكى دو دست داند دارند، قرار دهد.

\section{تتيجهيَيرى نهايى}

با اتوجه به نتايج تحقيقات قبلى كه نشان داده اند مهارت

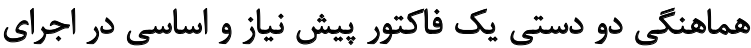

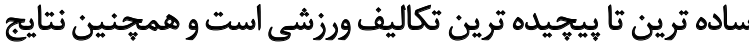

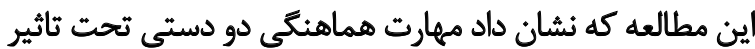

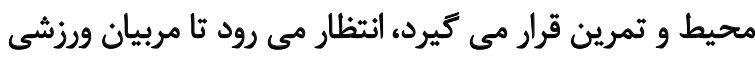

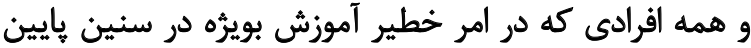

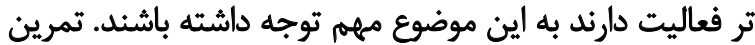

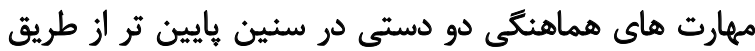

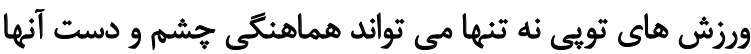

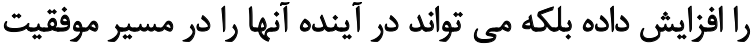

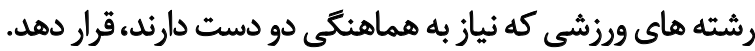

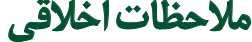

ييروى أز اصول اخلاق بئوهش

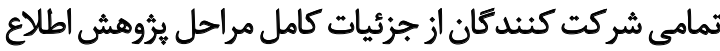

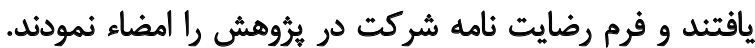

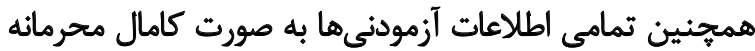

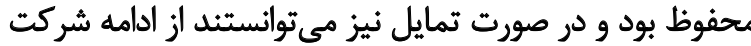

دريثروهش انصراف دهيند.

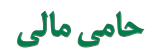

اين مقاله از يروزه تحقيقاتى دكتر ياسر خنجرى و دكتر الهر وريه

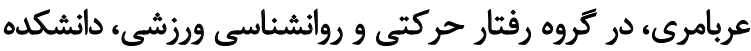

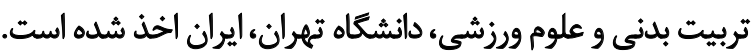

$$
\text { مشاركت نويسند مَّان }
$$

تمامى نويسندكان در انجام و نكارش مطالعه حاضر، به ميزان

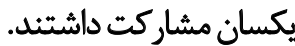

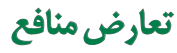

بنابر اظهار نويسندكان اين مقاله تعارض منافع ندارد

$$
\text { تشكر و قدرداثى }
$$

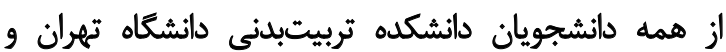

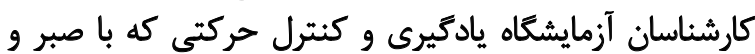

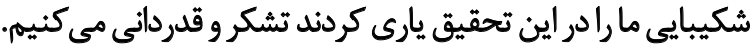




\section{References}

[1] Otte E, van Mier HI. Bimanual interference in children performing dual motor task. Hum Move Sci. 2006; 25(4-5):678-93. [DOI:10.1016/j. humov.2006.07.008] [PMID]

[2] Byblow WD, Summers JJ, Semjen A, Wuyts IJ, Carson RG. Spontaneous and intentional pattern switching in a multisegmental bimanual coordination task. Motor Control. 1999; 3(4):372-93. [DOI:10.1123/ mcj.3.4.372] [PMID]

[3] Swinnen SP, De Pooter A, Delrue S. Moving away from the in-phase attractor during bimanual oscillations. Studies in Percep Action. 1991; $315-9$.

[4] Swinnen SP. Intermanual coordination: From behavioural principle to neural-network interactions. Nat Rev Neurosci. 2002; 3(5):348-59. [DOI:10.1038/nrn807] [PMID]

[5] Wenderoth N, Puttemans V, Vangheluwe S, Swinnen SP. Bimanual training reduces spatial interference. J Mot Behav. 2003; 35(3):296-308. [DOI:10.1080/00222890309602142] [PMID]

[6] Swinnen SP, Dounskaia N, Duysens J. Patterns of bimanual interference reveal movement encoding within a radial egocentric reference frame. J Cogn Neurosci. 2002; 14(3):463-71. [DOI:10.1162/089892902317361 976] [PMID]

[7] Wiesendanger M, Rouiller EM., Kazennikov O, Perrig S. Is the supplementary motor area a bilaterally organized system? Adv Neurol. 1996; 70:85-93. https://pascal-francis.inist.fr/vibad/index.php?action=getRec ordDetail\&idt=3103296

[8] Jäncke L, Peters M, Himmelbach M, Nösselt T, Shah J, Steinmetz H. fMR study of bimanual coordination. Neuropsychologia. 2000; 38(2):164-74 [DOI:10.1016/S0028-3932(99)00062-7] [PMID]

[9] Toyokura M, Muro I, Komiya T, Obara M. Relation of bimanual coordination to activation in the sensorimotor cortex and supplementary motor area: Analysis using functional magnetic resonance imaging. Brain Res Bull. 1999; 48(2):211-7. [DOI:10.1016/S0361-9230(98)00165-8] [PMID]

[10] Kazennikov O, Hyland B, Corboz M, Babalian A, Rouiller E, Wiesendanger $M$. Neural activity of supplementary and primary motor areas in monkeys and its relation to bimanual and unimanual movement sequences. Neuroscience. 1999; 89(3):661-74. [DOI:10.1016/S03064522(98)00348-0] [PMID]

[11] de Oliveira SC. The neuronal basis of bimanual coordination: Recent neurophysiological evidence and functional models. Acta Psychol. 2002 110(2-3):139-59. [DOI:10.1016/S0001-6918(02)00031-8] [PMID]

[12] Schmidt RA. A schema theory of discrete motor skill learning. Psychol Rev. 1975; 82(4):225-60. [DOI:10.1037/h0076770]

[13] Schmidt RA, Zelaznik H, Hawkins B, Frank JS, Quinn Jr JT. Motor-output variability: A theory for the accuracy of rapid motor acts. Psychol Rev. 1979; 86(5):415-51. [DOI:10.1037/0033-295X.86.5.415]

[14] Marteniuk RG, MacKenzie CL, Baba DM. Bimanual Movement Control: Information processing in movement organization and execution. Atten Performance. 1980; 36(2):335-65. [DOI:10.1080/14640748408402163]

[15] Turvey MT. Preliminaries to a theory of action with reference to vision Perceiving, Acting Knowing. 1977; 2:211-65. http://www.haskins.yale. edu/Reprints/HL0168a.pdf

[16] Kugler PN, Kelso JS, Turvey M. On the concept of coordinative structures as dissipative structures: I. Theoretical lines of convergence. Tutor Mot Behav. 1980; 1:3-47. [DOI:10.1016/S0166-4115(08)61936-6]
[17] Antropomotorics JR. Theory of human motorics. Warszawa: Medical publishing PZWL; 2010

[18] Lin CH, Chou LW, Wei SH, Lieu FK, Chiang SL, Sung WH. Influence of aging on bimanual coordination control. Exp Gerontol. 2014; 53:40-7. [DOI:10.1016/j.exger.2014.02.005] [PMID]

[19] Wang C, Shea CH. Bimanual control strategies. Q J Exp Psychol. 2019; 72(4):966-78. [DOI:10.1177/1747021818781772] [PMID]

[20] Mason AH, Bruyn JL. Manual asymmetries in bimanual prehension tasks: Manipulation of object size and object distance. Hum Mov Sci. 2009; 28(1):48-73. [DOI:10.1016/j.humov.2008.09.002] [PMID]

[21] Riek S, Tresilian JR, Mon-Williams M, Coppard VL, Carson RG. Bimanua aiming and overt attention: One law for two hands. Exp Brain Res. 2003 153(1):59-75. [DOI:10.1007/s00221-003-1581-7] [PMID]

[22] Olivier I, Hay L, Bard C, Fleury M. Age-related differences in the reaching and grasping coordination in children: Unimanual and bimanual tasks. Exp Brain Res. 2007; 179(1):17-27. [DOI:10.1007/s00221-0060762-6] [PMID]

[23] Granek JA, Gorbet DJ, Sergio LE. Extensive video-game experience alters cortical networks for complex visuomotor transformations. Cortex 2010; 46(9):1165-77. [DOI:10.1016/j.cortex.2009.10.009] [PMID]

[24] Albines D. Sex-and Experience-Related Differences in Bimanual Coordination Development [MSc. thesis]. Toronto: York University; 2014. https://yorkspace.library.yorku.ca/xmlui/bitstream/handle/10315/28281/ Albines David 2014 Masters.pdf?sequence=2\&isAllowed=y

[25] Poole JL, Burtner PA, Torres TA, McMullen CK, Markham A, Marcum $\mathrm{ML}$, et al. Measuring dexterity in children using the Nine-hole Peg Test. $J$ Hand Ther. 2005; 18(3):348-51. [DOI:10.1197/j.jht.2005.04.003] [PMID]

[26] Loftesnes J, Sigmundsson H, Ingvaldsen R. Sex differences in bimanual coordination. Nordisk Fysioterapi. 2004; 8(1):43-7.

[27] Amirjani N, Ashworth NL, Gordon T, Edwards DC, Chan KM. Normative values and the effects of age, gender, and handedness on the Moberg Pick-Up test. Muscle Nerve. 2007; 35(6):788-92. [DOI:10.1002/ mus.20750] [PMID]

[28] Cohen NR, Pomplun M, Gold BJ, Sekuler R. Sex differences in the acquisition of complex skilled movements. Exp Brain Res. 2010; 205(2):183 93. [DOI:10.1007/s00221-010-2351-y] [PMID]

[29] Chraif M, Anitei M. Gender differences in motor coordination at young students at psychology. Int J Social Sci Humanity. 2013; 3(2):147-50. [DOI:10.7763/IJSSH.2013.V3.215]

[30] Allen LS, Richey MF, Chai YM, Gorski RA. Sex differences in the corpus callosum of the living human being. J Neurosci. 1991; 11(4):933-42. [DOI:10.1523/JNEUROSCI.11-04-00933.1991] [PMID] [PMCID]

[31] Barral J, Debu B, Rival C. Developmental changes in unimanual and bimanual aiming movements. Dev Neuropsychol. 2006; 29(3):415-29. [DOI:10.1207/s15326942dn2903_2] [PMID]

[32] Giedd JN, Rumsey JM, Castellanos FX, Rajapakse JC, Kaysen D, Vaituzis $A C$, et al. A quantitative MRI study of the corpus callosum in children and adolescents. Dev Brain Res. 1996; 91(2):274-80. [DOI:10.1016/01653806(95)00193-X] [PMID]

[33] Junaid KA, Fellowes S. Gender differences in the attainment of motor skills on the movement assessment battery for children. Phys Occup Ther Pediatr. 2006; 26(1-2):5-11. [PMID] 
[34] Flatters I, Hill ப, Williams JH, Barber SE, Mon-Williams M. Manual control age and sex differences in 4 to 11 year old children. PloS One. 2014; 9(2):e88692. [DOI:10.1371/journal.pone.0088692] [PMID] [PMCID]

[35] Fujii S, Kudo K, Ohtsuki T, Oda S. Intrinsic constraint of asymmetry acting as a control parameter on rapid, rhythmic bimanual coordination: A study of professional drummers and nondrummers. J Neurophysiol. 2010; 104(4):2178-86. [DOI:10.1152/jn.00882.2009] [PMID] [PMCID]

[36] Jäncke L, Shah N, Peters M. Cortical activations in primary and secondary motor areas for complex bimanual movements in professional pianists. Cog Brain Res. 2000; 10(1-2):177-83. [DOI:10.1016/S09266410(00)00028-8

[37] Doustan M, Boveiri K, Zilaei Bouri M, Seyfooriyan M. [The study of transfer of asymmetrical bimanual movement to its converse pattern: Analysis on bimanual movements theories (Persian)]. Motor Behav Sport Psychol. 2012; 8:553-64. http://ensani.ir/file/download/article/20161109135722-9761-212.pdf

[38] Moradi A. [The effect of increasing age and volleyball experience on continuous bilateral coordination task (Persian)]. J sport Sci. 2011; 2(5):5-18. https://www.sid.ir/fa/journal/ViewPaper.aspx?id=205993

[39] Carroll TJ, Benjamin B, Stephan R, Carson RG. Resistance training enhances the stability of sensorimotor coordination. Proc Biol Sci. 2001; 268(1464):221-7. [DOI:10.1098/rspb.2000.1356] [PMID] [PMCID]

[40] Khanjari Y, Tahmasebi S. [Investigating the differences in peripheral visual perception in ballplayers and non-ballplayers: Emphasis on the maturational perspective (Persian)]. Motor and Behaviour Sciences. 2019; 2(2):126-36. http://www.jmbs.ir/article 88230 937ea8ad33c58 dd25acb76f7a1225b24.pdf

[41] Sahan A, Erman KA. The effect of the tennis technical training on coordination characterictics. Open Sports Med J. 2009; 3:59-65. [DOI:10.217 4/1874387000903010059]

[42] Roh M. A comparison of head-hand coordination patterns during squash forehand strokes in expert and less-skilled squash players. Korean J Sport Biomech. 2018; 28(2):109-17. [DOI:10.5103/ KJSB.2018.28.2.109]

[43] Srinivasan D. Visuomotor coordination in symmetric and asymmetric bimanual reaching tasks [PhD. dissertation]. Michigan: University of Michigan; 2010

[44] Johansson RS, Westling G, Bäckström A, Flanagan JR. Eye-hand coordination in object manipulation. J Neurosci. 2001; 21(17):6917-32. [DOI:10.1523/JNEUROSCI.21-17-06917.2001] [PMID] [PMCID] 
This Page Intentionally Left Blank 